\title{
Etude de la Variabilité Morphologique de Saba Senegalensis (A. DC.) Pichon en Casamance (Sénégal)
}

\author{
Paul Diouf, \\ Siré Diedhiou, PhD \\ Arfang Ousmane Kémo Goudiaby,
}

Université Assane SECK de Ziguinchor, Faculté des Sciences et

Technologies, Laboratoire d'Agroforesterie et d'Ecologie,

Département d'Agroforesterie, Sénégal

Ibrahima Ndoye, Professeur Titulaire des Universités

Université Cheikh Anta Diop de Dakar, Faculté des Sciences et

Technologies, Département de Biologie Végétale, Sénégal

\section{Bothié Koita, PhD}

Institut Sénégalais de Recherches Agricoles,

Centre de Recherches zootehniques de Kolda, Sénégal

Doi: 10.19044/esj.2019.v15n9p500～URL:http://dx.doi.org/10.19044/esj.2019.v15n9p500

\section{Résumé}

Cette étude contribue à une meilleure connaissance de l'espèce Saba senegalensis, à travers une analyse de sa variabilité morphologique selon les zones de production en Casamance. Elle est réalisée dans six (06) sites à fort potentiel de production où des prélèvements de feuilles et de fruits ont été effectués sur 150 individus. Treize (13) descripteurs morphologiques ont été retenus. Les populations de Diembéring et de Nyassia présentent un développent foliaire plus important. Il est défini par des feuilles $(13,69 \mathrm{~cm})$ et des pétioles $(1,36 \mathrm{~cm})$ plus longs à Diembéring, et des feuilles relativement larges $(6,26 \mathrm{~cm})$ à Nyassia. Au niveau des sites de Thionk Essyl, Saré Bocar et Saré Sara, l'espèce Saba sengalensis est caractérisée par des feuilles moins larges, avec une moyenne de $5,77 \mathrm{~cm}$. Pour les descripteurs morphologiques relatifs aux fruits et graines, Nyassia et Thionk Essyl présentent un meilleur développement. Il ressort des analyses que, la variabilité des descripteurs morphologiques étudiées suit l'origine géographique mais pour certains descripteurs relatifs aux feuilles, leur variabilité serait liée aussi au génome.

Mots-clés: Saba senegalensis, fruits, feuilles, variabilité morphologique, Casamance 


\title{
Study of the Morphological Variability of Saba Senegalensis (A. DC.) Pichon in Casamance (Senegal)
}

\author{
Paul Diouf, \\ Siré Diedhiou, PhD \\ Arfang Ousmane Kémo Goudiaby,
}

Université Assane SECK de Ziguinchor, Faculté des Sciences et

Technologies, Laboratoire d'Agroforesterie et d'Ecologie,

Département d'Agroforesterie, Sénégal

Ibrahima Ndoye, Professeur Titulaire des Universités

Université Cheikh Anta Diop de Dakar, Faculté des Sciences et

Technologies, Département de Biologie Végétale, Sénégal

\section{Bothié Koita, PhD}

Institut Sénégalais de Recherches Agricoles,

Centre de Recherches zootehniques de Kolda, Sénégal

\begin{abstract}
This study was carried out to better understand the distribution of Saba senegalensis, through an analysis of its morphological variability in different areas of production in Casamance. Leaf and fruit samples were taken from 150 individuals across six (06) sites with high yield. Thirteen (13) morphological descriptors were used. Diembéring, Nyassia and Badiatte individual have a more important foliar development. It is defined by longer leaves $(13.69 \mathrm{~cm})$ and longer petioles $(1.36 \mathrm{~cm})$ in Diembering and broad leaves $(6.26 \mathrm{~cm})$ with short petioles $(1.08 \mathrm{~cm})$ inNyassia. InThionk Essyl, SaréBocar and Saré Sara, S. senegalensis individuals are characterized by smaller leaves with $5.77 \mathrm{~cm}$ on average. For morphological descriptors related to fruits and seeds, Nyassia and Thionk Essyl show better fruit development. From the results, it appears that the variability of the morphological descriptors studied follows the geographical origin but for some descriptors related to the leaves, their variability is also linked to the genome.
\end{abstract}

Keywords: Saba senegalensis, fruits, leaves, morphological variability, Casamance 


\section{Introduction}

L'arbre constitue une partie intégrante de la vie socioculturelle des peuples africains. Il joue un rôle appréciable dans l'économie et la qualité de vie des populations rurales et urbaines. En Casamance, région sud du Sénégal, les fruitiers sauvages tels que Saba senegalensis, constituent pour les populations, une source de revenus monétaires non négligeables, notamment en période de soudure, et assurent dans certains cas, une stabilité économique des ménages (Diop, 1997). L'ampleur du potentiel économique et alimentaire de l'espèce, justifie aujourd'hui la forte demande du marché sénégalais de son fruit, constituant ainsi la principale cause de la forte pression anthropique faite sur cette plante. Ceci justifie aujourd'hui le besoin de sa valorisation et de sa conservation durable. De facto, l'utilisation durable et la gestion d'une espèce fruitière, demandent une caractérisation de son matériel végétal. Dès lors, il apparait une nécessité d'élargir l'éventail d'informations sur les populations de S. senegalensis en Casamance. Selon Mars et Marrakchi (2000) cité par Kouaté (2010), la variabilité des espèces végétales, en général, s'exprime au niveau des caractéristiques de l'appareil végétatif et/ou de l'appareil reproducteur de la plante. Zhang (2002) considère que la description est nécessaire pour l'ensemble des activités d'amélioration génétique et de sélection variétale des plantes, car elle permet de cibler les descripteurs morphologiques intéressants et de connaître ceux qui sont liés aux facteurs environnementaux. En effet, l'existence de différents écotypes au sein d'une même espèce (au Sahel et dans le monde entier) aboutit à la mise en place de vastes programmes tels que la domestication. Pour une meilleure compréhension de la distribution de l'espèce, cette étude se propose de faire une analyse de la variabilité morphologique des populations de $S$. Senegalensis en Casamance dans six villages. L'hypothèse selon laquelle les populations de $S$. senegalensis sont différentes sur le plan morphologique, selon leur appartenance aux zones agro-écologique ou climatique, a été avancée.

\section{Matériel et Methodes}

\section{Zone d'étude et choix des sites}

La zone d'étude est localisée en Casamance dans deux domaines climatiques que sont le sud-soudanien côtier et le sud-soudanien continental (Sagna, 2005).Ces zones sont caractérisées par une forte humidité relative moyenne annuelle qui est liée à une assez longue présence de la mousson et de fortes précipitations par rapport au reste du pays. Entre 1985 et 2016, la pluviométrie moyenne annuelle est de $1054 \mathrm{~mm}$ (Ndiaye et al., 2019). Des sites de collection sont retenus dans six (06) villages: Thionk Essyl, Badiatte, Nyassia, Diémbéring, appartenant à la zone sud-soudanienne côtière et Sara 
Saré et Sara Bocar, localisés dans la zone sud-soudanienne continental (Figure1).

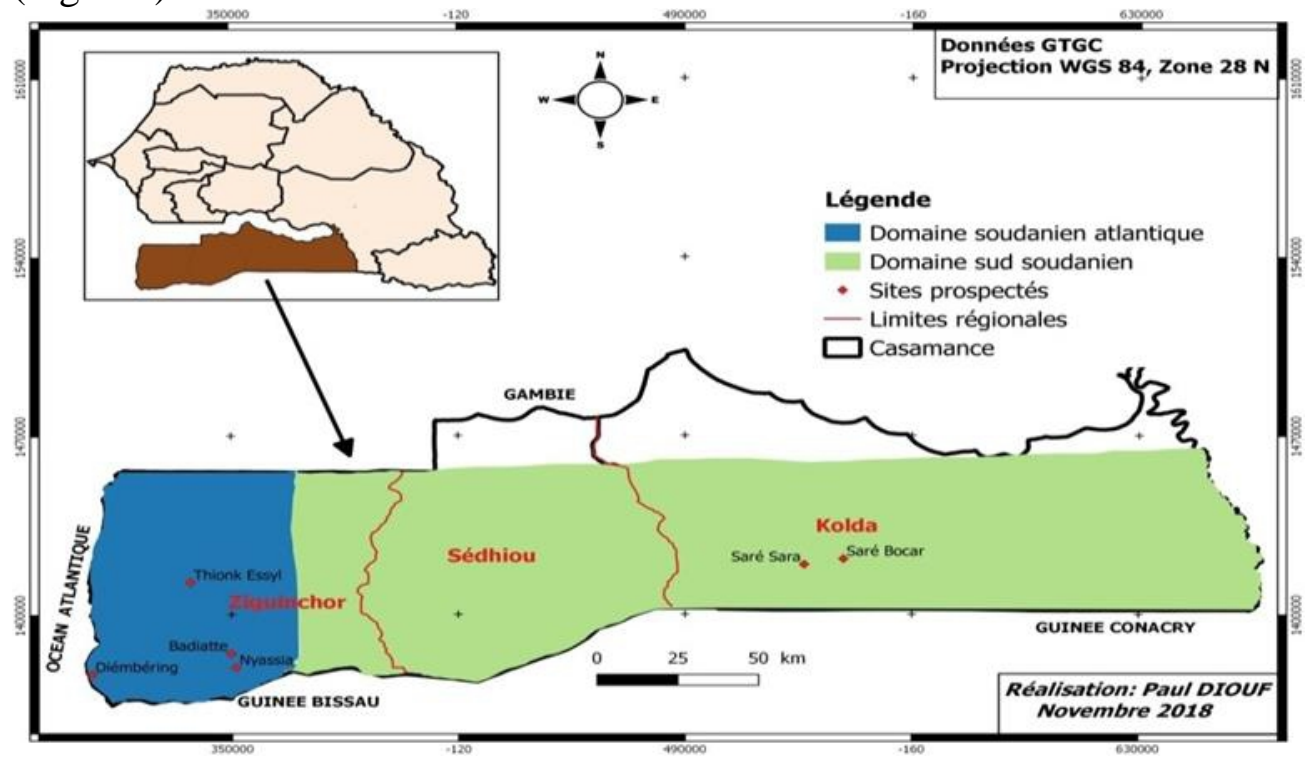

Figure 1: Carte de localisation des sites selon les zones climatiques

Par ailleurs, notre zone d'étude, selon l'approche agro-écologique, couvre deux(02) entités géographiques : la Basse et la Haute Casamance, avec des sols à texture multiple (Tableau 1). Ce choix des sites est la résultante d'études diagnostiques et d'observations montrant une bonne présence de populations de S. Senegalensis dans les formations végétales naturelles qui ceinturent ces villages; une population étant définie comme l'ensemble de tous les individus ou entités à partir desquels l'échantillonnage est conçu (Mariott, 1990). Doligez (1996) la définit comme étant, soit l'ensemble des arbres d'une même espèce situés sur une surface donnée, soit un groupe d'arbres de la même espèce géographiquement isolé des autres arbres de la même espèce.

Tableau 1 : Position géographique des sites et texture du sol

\begin{tabular}{|c|c|c|c|c|c|}
\hline \multirow{2}{*}{ Sites } & \multirow{2}{*}{ Entités géograpgiques } & \multirow{2}{*}{ Texture du sol } & \multicolumn{3}{|c|}{ Coordonnées géographiques } \\
\cline { 4 - 6 } & & & Longitude & Latitude & Altitude (m) \\
\hline Nyassia & Basse Casamance & sablo-limoneux & 351245 & 1379315 & 18 \\
\hline Badiatte & Basse Casamance & sablo-limoneux & 349661 & 1384774 & 19 \\
\hline Saré Sara & Haute Csamance & sablo-limono-argileux & 526736 & 1419453 & 21 \\
\hline Diémbéring & Basse Casamance & sablo-limoneux & 306721 & 1376684 & 14 \\
\hline Saré Bocar & Haute Csamance & limono-argilo-sableux & 538850 & 1421646 & 22 \\
\hline Thionk Essyl & Basse Casamance & limono-sableux & 337249 & 1412622 & 19 \\
\hline
\end{tabular}




\section{Méthodes d'étude}

Sur chaque site, des individus en cours de production de fruits ont été ciblés. vingt-cinq (25) individus par sites ont été retenus pour les prélèvements des échantillons. Pour chaque population étudiée, la distance minimale entre les individus sélectionnés est de 20 mètres. Cette approche permet de ne pas récolter du matériel sur des individus proches physiquement et génétiquement (Kouyaté, 2005). Les individus ont été géographiquement référencés à l'aide d'un GPS modèle 72 garmin. Au Total, 150 individus ont été suivis. Les prélèvements et mesures ont concerné les fruits et les feuilles; ils ont été effectués sur deux années successives. En raison de l'aspect sarmenteux des individus, leur hauteur, diamètre des tiges et des houppiers (qui constituent des descripteurs aussi intéressants dans la caractérisation d'une espèce), n'ont pu être mesurés.

\section{Descripteurs morphologiques \\ Descripteurs morphologiques des feuilles}

Des feuilles intactes et non parasitées ont été récoltées et mesurées à l'aide d'une règle graduée. Sur chaque individu, 10 feuilles ont été prélevées, soit 1500 unités mesurées. Les descripteurs qui ont été retenus sont la longueur des feuilles (Lfe), la largeur des feuilles (lafe) et la longueur du pétiole (Lp). La longueur est mesurée à partir du point d'insertion du pétiole (partie basale du limbe) jusqu'à l'extrémité apicale du limbe (Figure 2). La largeur est prise à partir de la moitié de la longueur du limbe suivant la méthode de Kouyaté (2005). La longueur des pétioles est prise depuis la base du pétiole jusqu'au point d'insertion du limbe. Ces mesures sont exprimées en $\mathrm{cm}$.

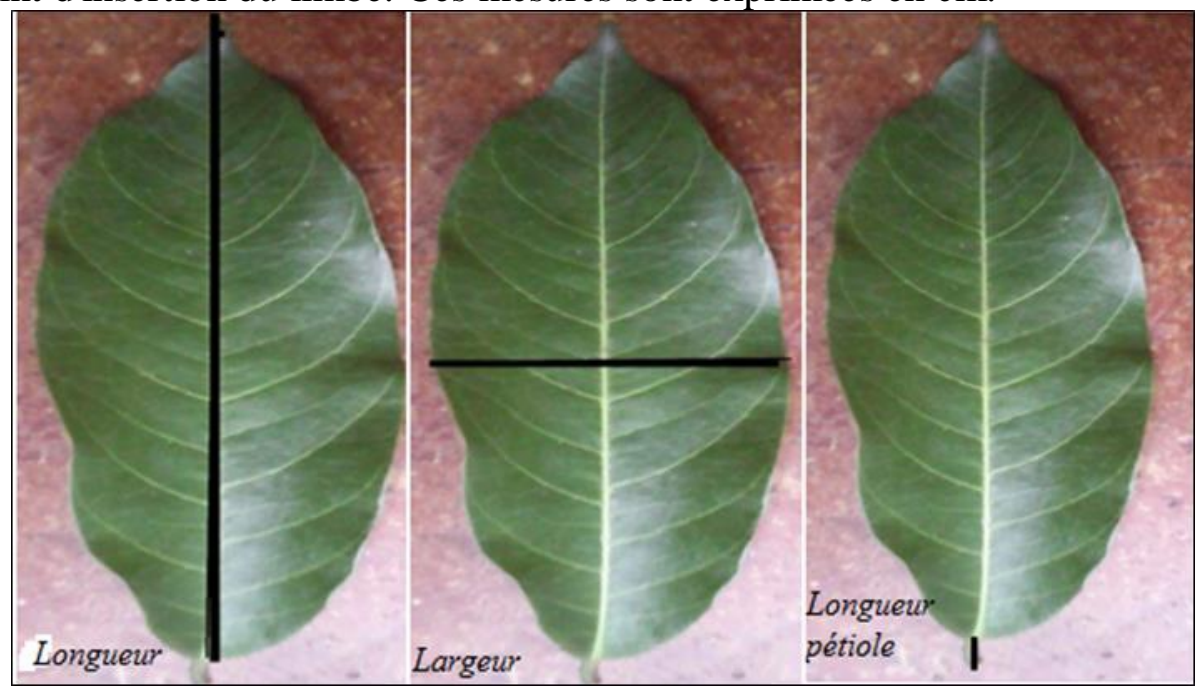

Figure 2: Mesures des feuillesde Saba senegalensis 


\section{Descripteurs morphologiques des fruits}

Les fruits ont été récoltés en utilisant une perche en bois surmonté d'un crochet en fer. Sur chaque arbre, dix (10) fruits ont été cueillis. La longueur (Lfr) et l'épaisseur (lafr) des fruits, exprimées en $\mathrm{cm}$, ont été mesurées à l'aide d'un pied à coulisse (Figure 3). La longueur est prise à partir du point d'attache du fruit au pédoncule jusqu'à l'extrémité basale. L'épaisseur est mesurée au niveau du plus grand diamètre du fruit. Le poids du fruit entier (Pfr) a été déterminé à l'aide d'une balance à précision $0.01 \mathrm{~g}$.

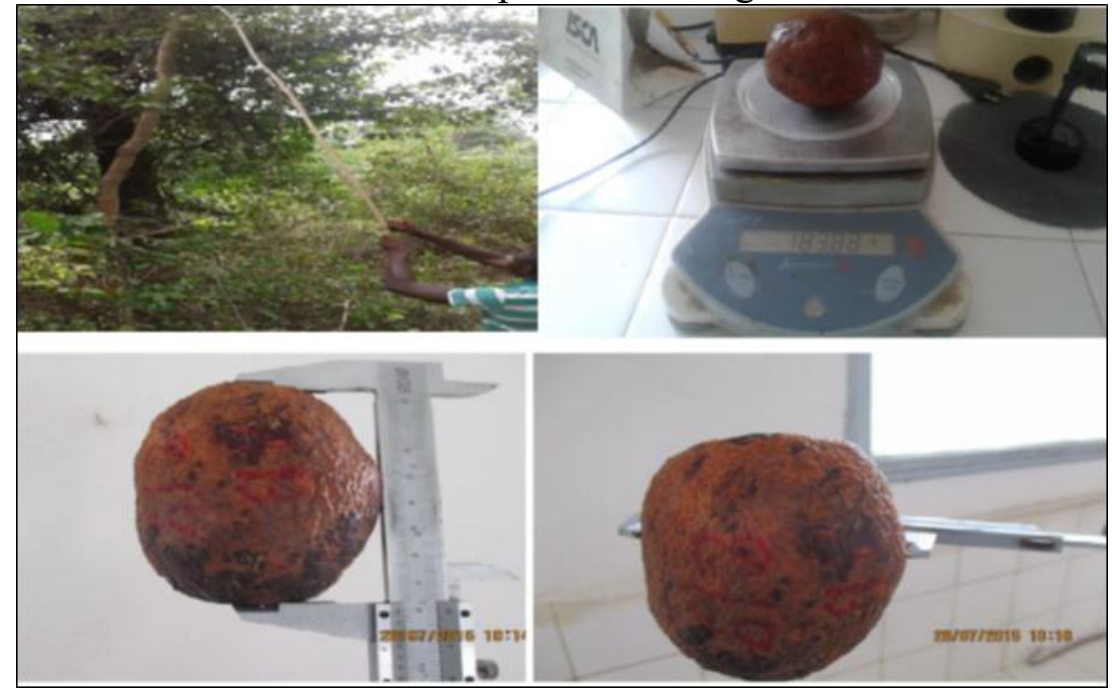

Figure 3: Récolte et mesures des fruits de Saba senegalensis

\section{Descripteurs morphologiques des graines et de la pulpe}

Les graines ont été extraites des fruits et conservées avec la pulpe dans une chambre froide, à une température de $-12^{\circ} \mathrm{C}$ pour celles qui ne pouvaient être mesurées dans l'immédiat. La longueur (Lg) et l'épaisseur (lag) des graines nues (sans pulpe) ont été mesurées à l'aide d'un pied à coulisse. L'épaisseur est mesurée au niveau du plus grand diamètre de la graine.

En plus des collectes de feuilles et de fruits, des prélèvements sol à 20 $\mathrm{cm}$ de profondeur ont été effectués et les paramètres géographiques tels que la longitude, la latitude et l'altitude des points de collecte, ont été relevés.

\section{Traitements des donnees}

A partir des meusres effectuées sur les fruits et les feuilles, des rapports sont calculés. Il s'agit du rapport entre la longueur et la largeur de la feuille (Qfe) et celui entre la longueur et la largeur du fruit (Qfr). De même, le rapport entre la longueur du pétiole et la longueur de la feuille (Qfp) a été déterminé.

La forme des feuilles et des fruits est déterminée selon la classification (tableau 2) utlisée par Clopton (2004). 
Tableau 2: Extrait de la table de nomenclature uniforme des formes planes à symétrie verticale sans marges rentrantes

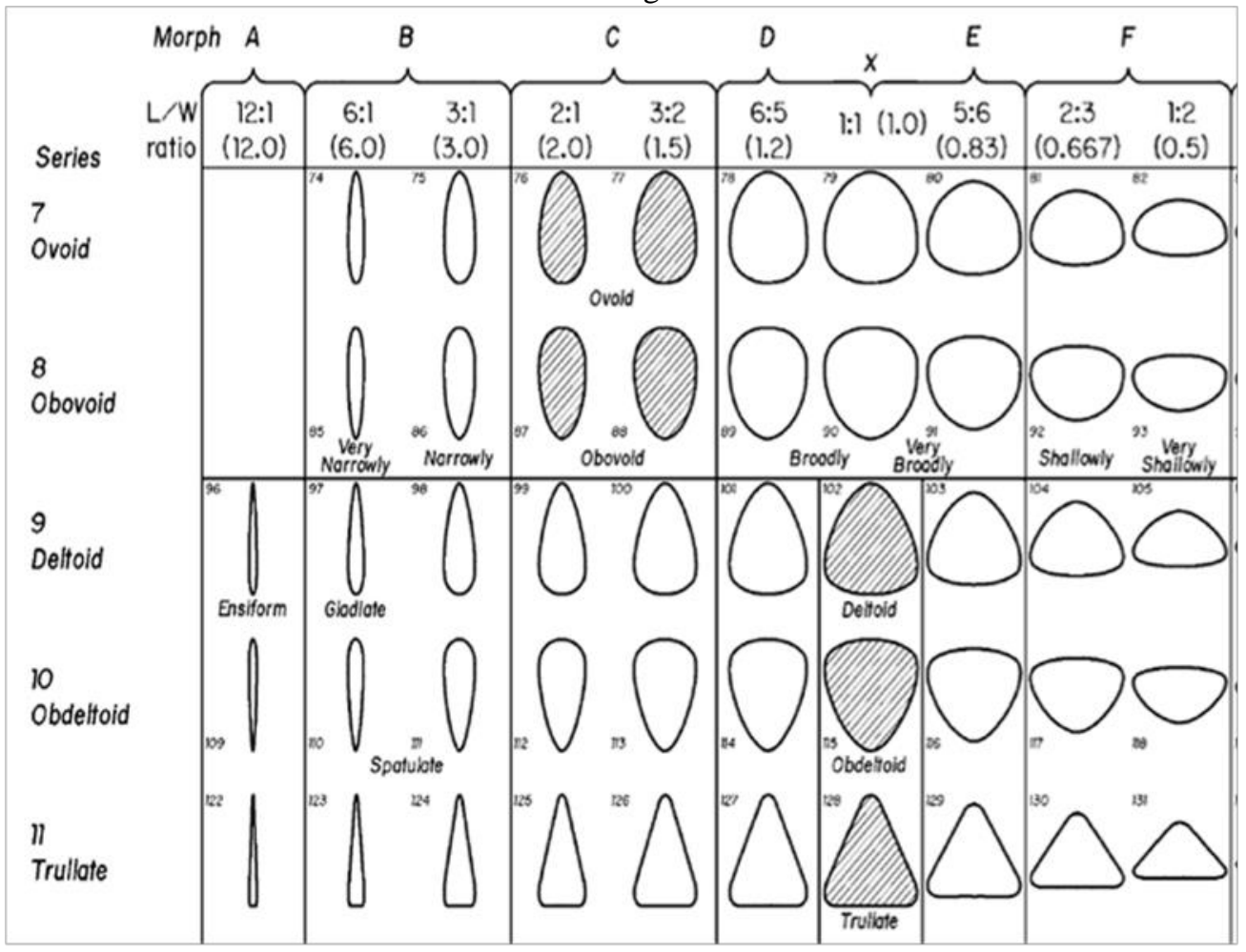

De même, pour les graines, le rapport entre la longueur et la largeur (Qg) a été déterminé. La pulpe qui entoure les graines est prélevée manuellement à l'aide d'un scalpel. Son poids $(\mathbf{P p})$, exprimé en g, est obtenu par déduction, selon la formule suivante :

\section{$\mathbf{P p}=\mathbf{P f r}-(\mathbf{P t g}+$ Ppéri $)$}

Avec Pfr = poids total du fruit ; Ptg = poids total des graines nues;Ppéri= poids du péricarpe

Le poids de la pulpe est ensuite, rapporté au poids total du fruit.

Au total, treize (13) descripteurs morphologiques ont été étudiés dont huit (08) sur 1500 fruits et cinq (05) sur 1500 feuilles, soit 19500 mesures effectuées. Pour chaque variable, la moyenne, l'écart type et le coefficient de variation (CV) sont calculés par population et pour l'ensemble des six (06) populations. La variabilité intra population et inter population est évaluée suivant l'échelle utilisée par Ouédraogo (1995):

$\mathrm{CV}=0-10 \%$ : variation faible

$\mathrm{CV}=10-15 \%$ : variation moyenne

$\mathrm{CV}=15-44 \%$ : variation assez importante

$(\mathrm{CV}>44 \%)$ : variation importante 
Des corrélations sont établies entre les descripteurs morphologiques et les facteurs géographiques, en faisant l'Analyse en Composantes Principales (ACP). Les analyses sont faites avec le logiciel XLSTAT.

\section{Resultats}

\section{Morphologie des feuilles de Saba senegalensis}

Les résultats des mesures des paramètres morphologiques des feuilles sont consignés dans le tableau 3.

Tableau 3 : Caractéristiques morphologiques des feuilles de Saba senegalensis

\begin{tabular}{|c|c|c|c|c|c|c|}
\hline \multirow[b]{2}{*}{ Localités } & \multirow[b]{2}{*}{ Paramétres statistiques } & \multicolumn{5}{|c|}{ Descripteurs morphologiques } \\
\hline & & $\mathrm{Lfe}(\mathrm{cm})$ & lafe $(\mathrm{cm})$ & Lpe (cm) & Qfe & Qfp \\
\hline \multirow{3}{*}{ Nyassia } & Moyenne & 13,60 & 6,26 & 1,08 & 2,17 & 12,82 \\
\hline & Ecartype & 1,45 & 0,62 & 0,16 & 0,26 & 2,36 \\
\hline & $\mathrm{CV}(\%)$ & 10,69 & 9,86 & 15,07 & 11,86 & 18,41 \\
\hline \multirow{3}{*}{ Saré Sara } & Moyenne & 13,21 & 5,91 & 1,15 & 2,23 & 11,57 \\
\hline & Ecartype & 1,44 & 0,58 & 0,12 & 0,33 & 1,66 \\
\hline & $\mathrm{CV}(\%)$ & 10,93 & 9,87 & 10,49 & 14,77 & 14,34 \\
\hline \multirow{3}{*}{ Thionk Essyl } & Moyenne & 12,88 & 5,68 & 1,13 & 2,26 & 11,57 \\
\hline & Ecartype & 1,30 & 0,57 & 0,14 & 0,28 & 1,78 \\
\hline & $\mathrm{CV}(\%)$ & 10,12 & 9,97 & 12,66 & 12,47 & 15,41 \\
\hline \multirow{3}{*}{ Saré Bocar } & Moyenne & 12,44 & 5,76 & 1,19 & 2,18 & 10,70 \\
\hline & Ecartype & 1,50 & 0,71 & 0,17 & 0,30 & 1,87 \\
\hline & $\mathrm{CV}(\%)$ & 12,04 & 12,30 & 14,39 & 13,65 & 17,50 \\
\hline \multirow{3}{*}{ Badiatte } & Moyenne & 13,49 & 6,20 & 1,23 & 2,19 & 11,23 \\
\hline & Ecartype & 1,55 & 0,69 & 0,17 & 0,24 & 2,29 \\
\hline & $\mathrm{CV}(\%)$ & 11,52 & 11,08 & 13,62 & 11,19 & 20,35 \\
\hline \multirow{3}{*}{ Diémbéring } & Moyenne $(\mathrm{cm})$ & 13,69 & 5,99 & 1,36 & 2,20 & 9,82 \\
\hline & Ecartype & 1,43 & 0,57 & 0,21 & 0,29 & 1,85 \\
\hline & $\mathrm{CV}(\%)$ & 12,44 & 9,50 & 15,47 & 13,16 & 18,82 \\
\hline \multicolumn{2}{|c|}{ Moyenne populations } & 13,11 & 5,96 & 1,18 & 2,21 & 11,35 \\
\hline \multicolumn{2}{|c|}{ Ecartype populations } & 1,53 & 0,66 & 0,18 & 0,29 & 3,39 \\
\hline \multicolumn{2}{|c|}{ CV (\%) population } & 11,63 & 11,05 & 15,52 & $13 ; 00$ & $29 ; 84$ \\
\hline
\end{tabular}

\section{Longueur des feuilles}

Les feuilles de $S$. senegalensis prélevées ont en moyenne une longueur de 13,11 cm. Les valeurs les plus importantes sont observées à Diembéring et à Nyassia avec respectivement une moyenne de13,69 $\mathrm{cm}$ et $13.60 \mathrm{~cm}$. A Saré Bocar, sont enregistrées les valeurs les moins importantes $(12,44 \mathrm{~cm})$. La variation entre les populations est moyenne (CV : 11, 63\%).

\section{Largeur des feuilles}

Les feuilles de S. Senegalensis en Casamance mesurent en moyenne $5,96 \mathrm{~cm}$ de largeur. Les individus de Nyassia et de Badiatte présentent les feuilles les plus larges avec respectivement $6,26 \mathrm{~cm}$ et $6,20 \mathrm{~cm}$. Les valeurs 
les moins importantes sont relevées à Thionk Essyl $(5,68 \mathrm{~cm})$. La variation inter-populationsest est moyenne $(\mathrm{CV}=11,05)$. A l'intérieur des populations, elle est moyenne à faible : le CV est compris entre 9,50 et $12,30 \%$.

\section{Longueur des pétioles}

Les pétioles des feuilles de $S$. senegalensis mesurent en moyenne 1,18 $\mathrm{cm}$. Cette longueur varie entre $1,08 \pm 0,16 \mathrm{~cm}$ à Nyassia et à $1,36 \pm 0,21 \mathrm{~cm}$ à Diembéring. La variation entre les populations est assez importante avec un CV égal à $15,52 \%$. La variation à l'intérieur des populations est moyenne à Saré Sara, Thionk Essyl, Sara Bocar et à Badiatte, avec un CV compris entre 10,49 et $14,39 \%$. Elle est assez importante à Nyassia $(\mathrm{CV}=15,07 \%)$ et à Diembéring $(\mathrm{CV}=15,47 \%)$.

\section{Rapport entre la longueur et la largeur des feuilles}

Le rapport moyen entre la longueur et la largeur des feuilles est de 2,21. Les valeurs les plus élevées sont notées à Saré Sara $(2,23)$ et à Thionk Essyl $(2,26)$. La variation inter populations est moyenne puisse que le CV est compris entre 10 et $15 \%$. S'agissant de la variation intra populations, elle est de même moyenne dans l'ensemble des sites; elle varie entre $11,19 \%$ à Badiatte et $14,77 \%$ à Saré Sara.

\section{Rapport entre la longueur des feuilles et la longueur des pétioles}

Les mesures effectuées lors de cette étude montrent que le rapport moyen entre la longueur des feuilles et la longueur des pétioles est de 11,35. La variation entre les populations est assez importante avec un CV compris entre 15 et $44 \%$. Au niveau intra populations, la variation est aussi assez importante dans tous les sites sauf à Saré Sara où elle est jugée moyenne avec un CV de $14,34 \%$.

\section{Morphologie des fruits et des graines}

Les résultats des mesures des paramètres morphologiques des fruits sont consignés dans le tableau 4. 
Tableau 4 : Caractéristiques morphologiques des fruits et graines de Saba senegalensis

\begin{tabular}{|c|c|c|c|c|c|c|c|c|c|}
\hline \multirow[b]{2}{*}{ Localités } & \multirow[b]{2}{*}{ Paramétres statistiques } & \multicolumn{8}{|c|}{ Descripteurs morp hologiques } \\
\hline & & $\operatorname{Pfr}(g)$ & $\operatorname{Lfr}(\mathrm{cm})$ & $\operatorname{lafr}(\mathrm{cm})$ & Qfr & $\operatorname{Lg}(\mathrm{cm})$ & $\operatorname{lag}(\mathrm{cm})$ & $Q g$ & Qpp \\
\hline \multirow{3}{*}{ Nyassia } & Moyenne & 173,66 & 8,01 & 6,86 & 1,17 & 1,92 & 1,24 & 1,54 & 0,34 \\
\hline & Ecartype & 54,55 & 0,97 & 0,76 & 0,09 & 0,13 & 0,06 & 0,19 & 0,08 \\
\hline & $\mathrm{CV}(\%)$ & 31,41 & 7,40 & 11,05 & 7,99 & 6,75 & 4,95 & 12,42 & 22,43 \\
\hline \multirow{3}{*}{ Saré Sara } & Moyenne & 141,14 & 7,30 & 6,18 & 1,18 & 1,72 & 1,11 & 1,55 & 0,40 \\
\hline & Ecartype & 37,63 & 0,82 & 0,67 & 0,10 & 0,12 & 0,06 & 0,09 & 0,13 \\
\hline & $\mathrm{CV}(\%)$ & 26,66 & 11,20 & 10,80 & 8,80 & 6,85 & 5,85 & 5,57 & 31,24 \\
\hline & Moyenne & 157,88 & 7,83 & 6,73 & 1,16 & 1,89 & 1,19 & 1,58 & 0,32 \\
\hline \multicolumn{2}{|c|}{ ThionkE ssy Ecartype } & 59,68 & 1,13 & 0,86 & 0,09 & 0,12 & 0,08 & 0,09 & 0,09 \\
\hline & $\mathrm{CV}(\%)$ & 37,80 & 14,38 & 12,82 & 8,14 & 6,25 & 6,85 & 5,67 & 28,66 \\
\hline \multirow{3}{*}{ Saré Bocar } & Moyenne & 159,62 & 7,38 & 6,32 & 1,16 & 1,88 & 1,23 & 1,53 & 0,32 \\
\hline & Ecartype & 58,80 & 1,02 & 0,90 & 0,13 & 0,11 & 0,07 & 0,10 & 0,08 \\
\hline & $\mathrm{CV}(\%)$ & 36,84 & 13,81 & 14,25 & 11,34 & 6,07 & 5,99 & 6,34 & 24,50 \\
\hline \multirow{3}{*}{ Badiatte } & Moyenne & 156,55 & 7,60 & 6,61 & 1,15 & 1,66 & 1,01 & 1,01 & 0,25 \\
\hline & Ecartype & 58,86 & 1,31 & 1,06 & 0,11 & 0,14 & 0,10 & 0,10 & 0,09 \\
\hline & $\mathrm{CV}(\%)$ & 37,60 & 17,17 & 16,06 & 9,18 & 8,54 & 9,48 & 9,48 & 34,87 \\
\hline \multirow{3}{*}{ Diémb éring } & Moyenne & 146,48 & 7,47 & 6,15 & 1,21 & 1,66 & 1,05 & 1,05 & 0,27 \\
\hline & Ecartype & 82,41 & 1,52 & 1,07 & 0,14 & 0,15 & 0,11 & 0,11 & 0,08 \\
\hline & $\mathrm{CV}(\%)$ & 56,26 & 20,39 & 17,35 & 11,14 & 9,08 & 10,45 & 10,45 & 27,63 \\
\hline \multicolumn{2}{|c|}{ Moyenne populations } & 160,08 & 7,63 & 6,5 & 1,17 & 1,79 & 1,14 & 1,38 & 0,31 \\
\hline \multirow{2}{*}{\multicolumn{2}{|c|}{ E cartype populations }} & 65,93 & 1,19 & 0,94 & 0,11 & 0,17 & 0,12 & 0,28 & 0,10 \\
\hline & CV (\%) population & 41,18 & 15,6 & 14,5 & 9,16 & 9,65 & 10,55 & 20,06 & 31,32 \\
\hline
\end{tabular}

\section{Le poids des fruits}

Les fruits de $S$. senegalensis pèsent en moyenne 158,61 g. Les valeurs les plus élevées sont enregistrées à Nyassia (173,66 g). Les populations de Saré Sara présentent les plus faibles valeurs, avec une moyenne de 141,14 g. $\mathrm{La}$ variation inter populations est assez importante, avec un $\mathrm{CV}$ égal à $41,18 \%$; il en est de même pour celle intra populations, hormis les populations de Diémbéring où le CV est de 56,26\%; ce qui traduit une variabilité importante.

\section{Longueur des fruits}

Les fruits de S. Senegalensis en Casamance mesurent 7,63 cm de long en moyenne. Les valeurs les plus élevées sont notées à Nyassia et à Thionk Essyl avec respectivement $8,01 \mathrm{~cm}$ et $7,83 \mathrm{~cm}$. La variation inter-populations est moyenne $(\mathrm{CV}=13,51)$. A l'intérieur des populations, la variation est faible $(\mathrm{CV}=7,40 \%)$ à Nyassia, moyenne à Saré Sara, Thionk Essyl et Saré Bocar $(\mathrm{CV}$ compris entre $11,20 \%$ et 14,38\%) et importante à Diembéring (20,39\%).

\section{Largeur des fruits}

Les fruits de $S$. senegalensis mesurent en moyenne $6,50 \mathrm{~cm}$ de large. La valeur maximale est enregistrée à Nyassia $(6,86 \mathrm{~cm})$. Les individus de Diembéring présentent les fruits les moins larges $(6,15 \mathrm{~cm})$. Nous notons une 
variation qui est moyenne entre les populations $(\mathrm{CV}=14,50 \%)$ et à l'intérieur des populations (CV compris entre $10,80 \%$ et 14,25\%) sauf à Diembéring où elle est assez importante avec un $\mathrm{CV}=17,35 \%$.

\section{Rapport entre la longueur et largeur des fruits}

A la lecture du tableau 3, il ressort que le rapport moyen entre la longueur et la largeur des fruits est de 1,17. Sur l'ensemble des sites étudiés, la variation intra-population est faible exceptés Sara Bocar et Diémbéring où elle est moyenne.

\section{Pulpe des fruits}

Les fruits de $S$. senegalensis renferment en moyenne 55,33g de pulpe. En moyenne, $34 \%$ du poids du fruit de S. Senegalensis est constitué de pulpe. Les valeurs les plus importantes sont relevées à Saré Sara $(\mathrm{Qpp}=0,40)$, suivi de Nyassia $(\mathrm{Qpp}=0,34)$. Les valeurs les moins importantes sont enregistrées à Badiatte. La variation inter-populations est assez importante (CV=27,74\%). Il en est de même à l'intérieur des populations étudiées : Nyassia $(C V=22,43 \%)$, Saré Sara $(\mathrm{CV}=31,24 \%)$, Thionk Essyl $(\mathrm{CV}=28,66)$ et Saré Bocar $(\mathrm{CV}=24,50)$.

\section{Longueur des graines}

Les mesures effectuées sur les graines des fruits de S. senegalensis montrent que celles-ci sont, en moyenne, longues de $1,79 \mathrm{~cm}$. Les graines enregistrées dans les populations de Thionk Essyl et Nyassia présentent les longueurs les plus élevées avec respectivement $1,92 \mathrm{~cm}$ et $1,89 \mathrm{~cm}$. Les écarts notés entre ces valeurs,dans les différents sites, ne sont pas aussi importantes puisque la variation inter-population, de même que celle intra-population est faible (CV comprise entre 6,07 et 9,65).

\section{Largeur des graines}

Les graines de $S$. senegalensis de la Casamance mesurent en moyenne 1,14 cm de large. Les valeurs les plus élevées sont enregistrées à Nyassia avec une moyenne de 1,24 cm. Celles de Badiatte sont moins larges. La variation inter-population est moyenne avec un CV compris entre 10 et $15 \%$.Cependant, la variation enregistrée à l'intérieur des populations est faible hormis celle de Diémbéring où le CV est égal à 10,45.

\section{Rapport entre la longueur et la largeur des graines}

Le rapport moyen entre la longueur et la largeur des graines est de 1,38. Les valeurs maximale et minimale sont respectivement observées à Thionk Essyl $(1,58)$ et à Badiatte $(1,01)$. La variation inter-population est assez importanteparce que la valeur du CV est comprise entre 15 et $44 \%$. Au niveau 
intra population, les variations vont de faibles à moyennes avec un CV compris entre $5,57 \%$ à Saré Sara et $12,42 \%$ à Nyassia.

\section{Corrélation entre les descripteurs morphologiques et les facteurs géographiques}

Il ressort de l'analyse du niveau de corrélation entre les descripteurs morphologiques eux-mêmes (tableau 4) que la longueur des feuilles est positivement corrélée à la longueur du pétiole, au rapport entre longueur feuille et largeur feuille et au rapport entre longueur feuille et longueur pétiole. Les coefficients de corrélation sont respectivement de 0,$521 ; 0,716$; et 0,446. Des corrélations positives sont aussi observées entre la longueur des fruits et la largeur des fruits $(r=0,745)$, entre la longueur de la feuille et le rapport entre la longueur et la largeur des fruits $(r=0,154)$. Par ailleurs des corrélations négatives sont notées entre la longueur de la feuille et la largeur de la graine. Le rapport entre la longueur et la largeur des graines sont également corrélés négativement. Ceci signifie que plus les feuilles sont longues moins les graines sont larges. De même, il a été noté une corrélation négative entre largeur de la graine et la longueur du pétiole $(r=0,199)$.

Tableau 4: matrice de corrélation entre les descripteurs morphologiques

\begin{tabular}{|c|c|c|c|c|c|c|c|c|c|c|c|c|c|}
\hline & Lfe & lafe & Lpe & Qfe & Qfp & Lfr & lafr & Pfr & Qfr & Lg & lag & $Q g$ & Qpp \\
\hline Lfe & 1 & 0,202 & 0,521 & 0,716 & 0,446 & 0,047 & 0,055 & 0,036 & 0,154 & 0,020 & $-0,067$ & $-0,072$ & 0,009 \\
\hline lafe & 0,202 & 1 & 0,097 & $\cdot 0,078$ & 0,110 & 0,032 & 0,044 & 0,043 & $-0,044$ & $-0,081$ & $-0,049$ & $-0,122$ & $-0,040$ \\
\hline Lpe & 0,521 & 0,097 & 1 & 0,483 & $-0,064$ & $-0,026$ & $-0,052$ & $-0,003$ & 0,245 & $-0,199$ & $-0,249$ & $-0,329$ & $-0,115$ \\
\hline Qfe & 0,716 & $-0,078$ & 0,483 & 1 & 0,435 & 0,028 & 0,014 & $-0,005$ & 0,217 & 0,106 & $-0,014$ & 0,028 & 0,037 \\
\hline Qfp & 0,446 & 0,110 & $-0,064$ & 0,435 & 1 & 0,014 & 0,034 & 0,000 & 0,042 & 0,144 & 0,095 & 0,112 & 0,056 \\
\hline Lfr & 0,047 & 0,032 & $-0,026$ & 0,028 & 0,014 & 1 & 0,745 & 0,733 & 0,202 & 0,097 & 0,090 & 0,033 & $-0,003$ \\
\hline lafr & 0,055 & 0,044 & $-0,052$ & 0,014 & 0,034 & 0,745 & 1 & 0,780 & $-0,003$ & 0,104 & 0,103 & 0,053 & $-0,049$ \\
\hline Pfr & 0,036 & 0,043 & $-0,003$ & $-0,005$ & 0,000 & 0,733 & 0,780 & 1 & 0,035 & 0,046 & 0,050 & $-0,034$ & $-0,050$ \\
\hline Qfr & 0,154 & $-0,044$ & 0,245 & 0,217 & 0,042 & 0,202 & $-0,003$ & 0,035 & 1 & 0,044 & $-0,037$ & $-0,083$ & $-0,011$ \\
\hline Lg & 0,020 & $-0,081$ & $-0,199$ & 0,106 & 0,144 & 0,097 & 0,104 & 0,046 & 0,044 & 1 & 0,613 & 0,560 & 0,131 \\
\hline lag & $-0,067$ & $-0,049$ & $-0,249$ & $-0,014$ & 0,095 & 0,090 & 0,103 & 0,050 & $-0,037$ & 0,613 & 1 & 0,643 & 0,155 \\
\hline Qg & $-0,072$ & $-0,122$ & $\cdot-0,329$ & 0,028 & 0,112 & 0,033 & 0,053 & $-0,034$ & $-0,083$ & 0,560 & 0,643 & 1 & 0,332 \\
\hline Qpp & 0,009 & $-0,040$ & $\cdot-0,115$ & 0,037 & 0,056 & $-0,003$ & $-0,049$ & $-0,050$ & $-0,011$ & 0,131 & 0,155 & 0,332 & 1 \\
\hline
\end{tabular}

En gras, valeurs significatives (hors diagonale) au seuil alpha $=0,050$ (test bilatéral)

Les résultats sur le niveau de corrélation entre les coordonnées géographiques et les variables morphologiques étudiées, sont consignés dans le tableau 5. Ces résultats montrent que la longueur et la largeur des feuilles et la longueur des pétioles sont négativement corrélées aux coordonnées géographiques. Ceci traduit une diminution des valeurs de ces descripteurs morphologiques avec l'augmentation de l'altitude, de la latitude et de la longitude. La longueur et la largeur des fruits sont négativement et faiblement corrélées à la longitude. En outre, une corrélation négative est observée entre le poids du fruit et la longitude. 
Tableau 5: Corrélation entre les descripteurs morphologiques et les facteursgéographiques

\begin{tabular}{lrrr}
\hline & Longitude & \multicolumn{1}{c}{ Latitude } & \multicolumn{1}{c}{ Altitude } \\
\hline Lfe & $-0,132$ & $-0,187$ & $-0,100$ \\
lafe & $-0,124$ & $-0,261$ & $-0,112$ \\
Lpe & $-0,101$ & $-0,145$ & $-0,282$ \\
Qfe & $-0,007$ & 0,055 & 0,003 \\
Qfp & $-0,038$ & $-0,042$ & 0,040 \\
Lfr & $-0,170$ & $-0,110$ & $-0,067$ \\
lafr & $-0,176$ & $-0,095$ & $-0,006$ \\
Pfr & $-0,096$ & $-0,092$ & 0,004 \\
Qfr & $-0,043$ & $-0,079$ & $-0,147$ \\
Lg & 0,093 & 0,195 & 0,264 \\
lag & 0,215 & 0,274 & 0,324 \\
Qg & 0,448 & 0,595 & 0,560 \\
Qpp & 0,291 & 0,261 & 0,238 \\
Longitude & 1 & 0,789 & 0,798 \\
Latitude & 0,789 & 1 & 0,799 \\
Altitude & 0,798 & 0,799 & 1 \\
\hline
\end{tabular}

En gras, valeurs significatives (hors diagonale) au seuil alpha $=0,050$ (test bilatéral)

\section{Importance des variables morphologiques selon les localités}

L'ensemble des variables morphologiques ont été regroupés pour une Analyse en Composantes principales (ACP). Cette ACP nous a permis d'obtenir la Figure 4, montrant que la longueur, la largeur et le poids des fruits, de même que la longueur et la largeur des graines et le rapport entre la longueur et la largeur des graines définissent l'axe F1. S'agissant de l'axe F2, la longueur des feuilles, la longueur des pétioles et le rapport entre la longueur et la largeur des feuilles sont les plus déterminants.

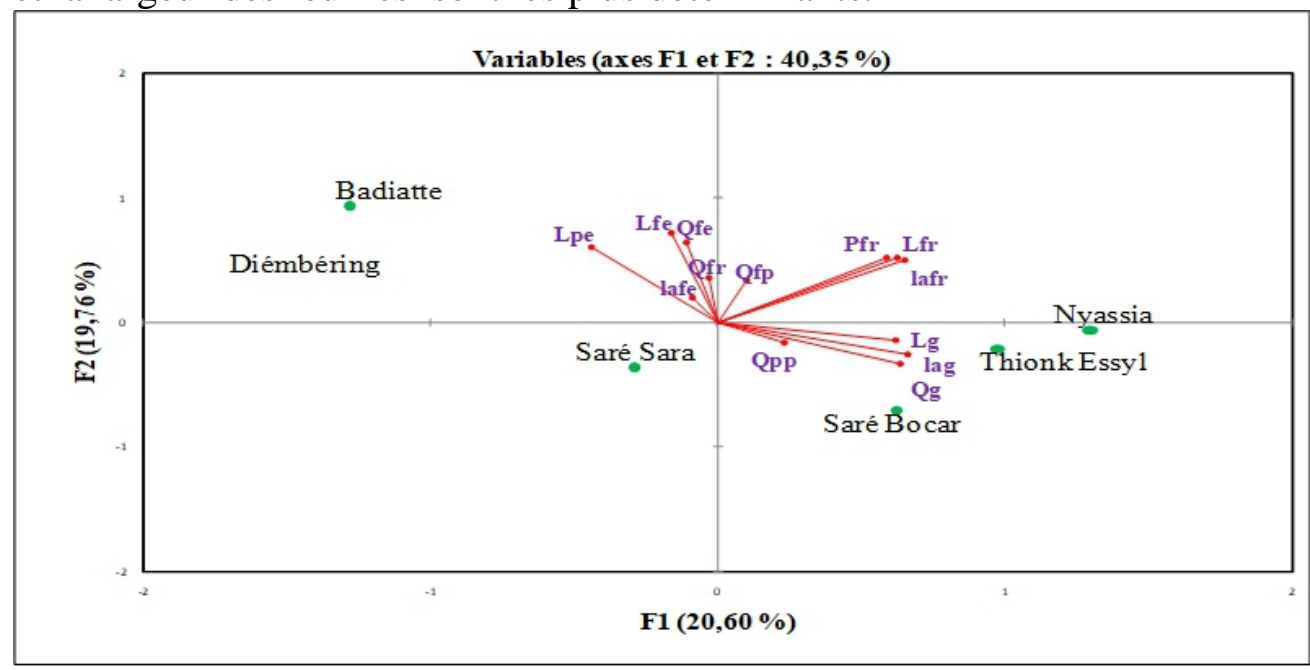

Figure 4: Répartition des sites en fonction des descripteurs morphologiques

Il ressort de ces résultas que les populations de Diembéring, Nyassia et de Badiatte ont un développent foliaire plus marqué. Celles de Diembéring sont caractérisées par des feuilles longues à pétioles longs tandis que celles de Nyassia présentent de larges feuilles mais à pétioles courts. Quant aux 
populations de Thionk Essyl, Saré Bocar et Saré Bocar, elles sont caractérisées par des feuilles moins développées. Les descripteurs morphologiques relatifs aux fruits sont mieux exprimés par les populations de Nyassia et de Thionk Essyl qui présentent les fruits les plus intéresssants en termes de longueur et largeur. En outre, dans ces deux sites, avec celui de Saré Bocar, sont enrégistrées les plus graines grosses.

\section{Discussion}

\section{Variation morphologique de feuilles}

Les mesures effectuées sur les feuilles de $S$. senegalensis montrent que celles-ci ont, en moyenne, une longueur de 13,11 cm et une largeur de 5,96 $\mathrm{cm}$. Ces résultats confirment ceux de Bérhaut (1967), selon qui, les feuilles de S. senegalensis sont longues de 8 à $15 \mathrm{~cm}$ et larges de 4 à $6 \mathrm{~cm}$. Dans sa description de l'espèce S. senegalensis, Arbonnier (2009) affirme que la longueur du pétiole est comprise entre 1 et $1,5 \mathrm{~cm}$; ce qui conforte les résultats de cette présente étude. Puisque les dimensions des pétioles dans les différentes populations, varient entre 1,06 et $1,38 \mathrm{~cm}$. Les résultats du rapport entre la longueur de la feuille et la largeur de la feuille montrent que celui-ci se trouve aux niveaux de 2:1 et 3:2 suivant la classification de Clopton (2004). Selon, cette classification, la feuille a une forme ovoïde à ovale avec un sommet acuminé. Toutefois, Bérhaut (1967) et Arbonnier (2009), en plus de cette forme ovale du limbe qu'ils ont décrite, mentionnent également une forme elliptique du limbe. Dans la présente étude, cette forme n'a pas été rencontrée.

Sur les descripteurs morphologiques foliaires (longueur et largeur feuilles, longueur pétiole), en dépit de leur corrélation (négative) avec les coordonnées géographiques, les variations inter-populations sont faibles à moyennes. La seule variation intra-population assez importante concerne la longueur du pétiole et elle est enregistrée à Diémbéring où, par ailleurs, les plus longs pétioles ont été notés. Cette variation peut être due à l'hétérogénéité topographique du site ; certains individus étant localisés à une basse altitude (à moins de $500 \mathrm{~m}$ du littoral). Ceci laisse supposée l'existence d'une stratégie développée par l'espèce pour mieux s'adapter aux conditions climatiques de cette zone, caractérisée par un climat soudano guinéen à sub-guinéen à empreinte côtière.

Les feuilles de Saba senegalensis relevées à Saré Bocar, Saré Sara et Thionk Essyl, ont des dimensiens (longueur et largeur) moins importantes par rapport à celles des trois autres localités. Saré Bocar et Saré Sara appartiennent au domaine climatique soudano guinéen continental; dans cette partie de la Haute Casamance, les sols sont formés de vastes plateaux développant des cuirasses affleurantes (Niane 1984). Ceci expliquerait le développement foliaire moindre des individus de S. Senegalensis dans ces zones. 
L'appartenance des populations de Thionk Essyl à ce groupe (développement foliaire moindre), laisse supposer que c'est liée aux génomes des individus de Saba senegalensis puisque Thionk Essyl est inclus dans le même domaine climatique que Diembéring, Nyassia et de Badiatte (Basse Casamance), où, en dehors des zones basses et humides, les sols sont caractéristiques d'affleurements de grès argileux (Niane, 1984).

\section{Variation morphologique des fruits}

Les mesures effectuées sur les fruits montrent que ceux-ci ont en moyenne une longueur de 7,63 cm et sont larges de 6,5 cm. Selon Bérhaut (1967) et Arbonnier (2009), les fruits de S. senegalensis sont longs de 7 à 10 $\mathrm{cm}$ et larges de 6 à $8 \mathrm{~cm}$. Les valeurs obtenues sont dans ces intervalles trouvés par ces auteurs. Par contre, des études réalisées par Traoré (2000) sur la même espèce dans le parc national de Niokolokoba (partie orientale du Sénégal) montrent une longueur moyenne de $20,75 \mathrm{~cm}$ et une largeur de $10,82 \mathrm{~cm} \mathrm{du}$ fruit. Ces chiffres sont deux (02) fois supérieurs à ceux trouvés dans la présente étude. Cet écart pourrait être lié aux conditions climatiques différentes des zones. Le parc national de Niokolo koba est situé dans la zone de Kédougou qui est l'une des plus pluvieuses du Sénégal avec au moins 1300 $\mathrm{mm} / \mathrm{an}$ et des températures généralement élevées avec des maxima variant entre $34^{\circ}$ et $42^{\circ}$ et une humidité relative très élevée qui dépasse $97 \%$ en hivernage (ANSD, 2010). De même, les travaux de Traoré (2000) ont montré que les fruits de Saba senegalensis dans cette zone, pèsent en moyenne $188 \mathrm{~g}$; ce qui est supérieur au poids moyen des fruits de la Casamance. Des variations assez importantes $(\mathrm{CV}=41,18 \%)$ inter-populations sur le poids du fruit ont été notées dans cette étude. Des mesures réalisées dans plusieurs zones du Sénégal sur Adansonia digitata L. (Bombacaceae) montrent, selon le poids des fruits, une variation inter-populations moyenne avec un CV égale à 11,4\% (Soloviev et al., 2004). Concernant les descripteurs longueur et largueur du fruit, les variations inter populations sont faibles. D'ailleurs la corrélation entre ces descripteurs et les coordonnées géographiques n'est pas assez forte. Cependant, les variations intra-populations qui vont de moyennes à assez fortes (cas de Badiatte et Diémbéring), seraient liées aux génomes. Le rapport entre la longueur et la largeur du fruit de $S$ senegalensis, suivant la classification de Clopton (2004), se trouve dans entre les niveaux 3:2 et 6:5. Ceci confirme la forme ovoïde du fruit décrite dans la littérature. Cependant les fruits enregistrés à Diémbéring présentent des formes ovoïdes etobovoïdes (la partie la plus large se rapproche du milieu). 


\section{Variation morphologique des graines}

Les résultats obtenus sur la longeur et la largeur de la graine corroborent ceux de Traoré (2000) qui affirme que la longueur moyenne des graines de $S$. senegalensis est de 1,5 cm et que la largeur moyenne est de 1,01 $\mathrm{cm}$. Le rapport entre la longueur et la largeur indique que la forme de la graine de S. Senegalensis est obovoïde. La variabilité des ces trois descripteurs (longueur, largeur et rapport entre longueur et largeur) varie de faible à moyenne entre les populations, bien que ceux-ci soient relativement corrélés aux coordonnées géographiques. Suivant l'ACP, ces variables, en plus de celles relatives aux fruits,définissent principalement l'axe F1. Ceci indique que les populations de Nyassia et de Thionk Essyl, appartenant à la même zone climatique (soudano guinéen côtier), renferment les valeurs spécifiques des graines les plus élevées. De même, celles de Saré Bocar qui sont incluses dans la zone soudano guinéen continental, ont des graines relativement développées. Il en ressort que ces descripteurs ne suivent pas seulement l'origine géographique mais pourraient être influencés aussi par le génome des individus de Saba senegalensis.

\section{Conclusion}

Les résultats ont permis de mettre en relief la variabilité morphologique des feuilles et des fruits des populations de Saba senegalensis en Casamance. Il ressort de cette étude que les feuilles de l'espèce sont oviformes et les fruits ont une forme ovoïde et obovoïde. Les graines ont quant à elles, une forme obovoïde. Les populations de Diembéring, de Nyassia et de Badiatte présentent un développent foliaire plus intéressant, défini par des feuilles longues et à pétioles longs à Diembéring et des feuilles larges à pétioles courts à Nyassia. A l'opposé, nous avons le groupe de populations composé par Thionk Essyl et Saré Bocar avec des feuilles moins développées. S'agissant des descripteurs morphologiques relatifs aux fruits et graines, Nyassia et Thionk Essyl présentent les fruits les plus intéressants selon la grosseur. Ces informations issues de milliers de mesures sur des fruits et feuilles de S. Senegalensis peuvent constituer un support pour les programmes d'amélioration, de production et de protection des fruitiers forestiers sauvages de la Casamance. La création de variétés, l'implantation de vergers de $S$. Senegalensis et la protection in situde l'espèce, pourraient participer d'avantage à booster la production pour ainsi satisfaire la demande nationale et par la même occasion, améliorer le niveau de vie des populations locales, premiers acteurs dans la filière. 


\section{References:}

1. ANSD (Agence Nationale des Statistiques Démographiques), 2010. Situation économique du Sénégal en 2009. Dakar, Sénégal, ISSN 0850-1491. $19 \mathrm{p}$.

2. Arbonnier M., 2009. Arbres, arbustes et lianes des zones sèches de l'Afrique de l'Ouest, Ed. Quae, Paris, ISBN MNHN 978-2-85653-626$1.577 \mathrm{p}$.

3. Berhaut J., 1967. Flore du Sénégal. 2ème Ed. Clairafrique, Dakar. 485 p.

4. Clopton R. E., 2004. Standard Nomenclature and Metrics of Plane Shapes for use in GregarineTaxonomy. Comp. Parasitol. 71(2), 2004. 130-140.

5. Diop G., 1997. Contribution à la connaissance des potentialités des jachères en espèces forestières ligneuses alimentaires et en espèces forestières ligneuses à usage médical dans le département de Bignona (Sénégal). Mémoire de fin d'étude ; Ecole Nationale du Génie Rural, des Eaux et des Forêts/France. 90 p.

6. Doligez A., 1996. Evolution de la diversité génétique intra-population et de sa structure. Etude d'un modèle de simulation spatialisé de la gestion des ressources génétiques forestières tropicales. Institut National Agronomique Paris-Grignon. CIRAD-Forêt. 273 p.

7. Kouyaté A. M. 2005. Aspects ethnobotaniques et étude de la variabilité morphologique, biochimique et phénologique de Detariummicrocarpum Guill. \&Perr. au Mali. Thèse de doctorat de $3^{\text {ième }}$ cycle. $190 \mathrm{p}$.

8. Mariott F. H. C., 1990. A dictionary of statistical terms. Fifthedition. Longman. $223 \mathrm{p}$

9. Mars M. etMarrakchi M., 2000. Etude de la variabilité intra-arbre chez le grenadier (PunicagranatumL.). Application à l'échantillonnage des fruits. Fruits. 347-355.

10. Ndiaye A., Mbaye T., Ngom D., Ly M. O., Diouf D., 2019. Fonctionnement Hydrique du Baobab (Adansonia digitata L.) en Moyenne et Haute Casamance (Sénégal). European Scientific Journal, Vol.15, No.3, January 2019. 292-312

11. Niane A. B., 1984. Etudes cartographiques et agro-pédologiques des sols de Plateau de Basse Casamance. Mémoire de confirmation ISRA. $98 \mathrm{p}$.

12. Ouédraogo A. S., 1995. Parkiabiglobosa (Fabaceae) en Afrique de l'Ouest. Biosystématique et amélioration. Thèse. Univ. agron. Wagening. Inst. For. Nat. Res. IBN-DLO. Netherlands. 205 p. 
13. Sagna P., 2005. Dynamique du climat et son évolution récente dans la partie ouest de l'Afrique occidentale. Thèse de doctorat d'état, Université Cheikh Anta Diop. 786 p.

14. Soloviev P., Niang T. D., Gaye A. et Totte A., 2004. Variabilité des caractères physicochimiques des fruits de trois espèces ligneuses de cueillette, récoltés au Sénégal. Adansonia digitata, Balanites aegyptiaca et Tamarindus indica. Fruits. 109-119.

15. Traoré A., 2000. Étude de la reproduction et du développementde Saba senegalensis (A. DC) Pichon au Sénégal. Thèse de Doctorat de $3^{\text {ème }}$ Cycle, Option Biologie Végétale, FST/UCAD, Dakar. 100 p.

16. Zhang D. 2002. Marqueurs moléculaires. Outils de choix pour le génotypage des plantes. In (eds.): Les apports de la biologie moléculaire en arboriculture fruitière. 12e colloque sur les recherches fruitières. 30-31 Mai 2002. Bordeaux. 3 p. 